\title{
The role of macrophage polarization on fibroblast behavior-an in vitro investigation on titanium surfaces
}

\author{
Xuzhu Wang ${ }^{1,2}$ - Yulan Wang ${ }^{1}$ Dieter D. Bosshardt ${ }^{3}$. Richard J. Miron ${ }^{4}$. \\ Yufeng Zhang ${ }^{1,2}$
}

Received: 12 July 2016 / Accepted: 23 June 2017 / Published online: 14 July 2017

(C) Springer-Verlag GmbH Germany 2017

\begin{abstract}
Objectives This study investigated the effect of smooth and rough titanium surface topographies on macrophage polarization and their influence on gingival fibroblast behavior cultured on titanium surfaces.

Materials and methods RAW 264.7 macrophages were seeded on smooth (pickled titanium (PT)) and rough Sand-blasted with Large grit particles followed by Acid-etching (SLA) titanium surfaces and first investigated for macrophage polarization towards tissue-inflammatory M1 macrophages or wound-healing M2 macrophages. Thereafter, culture media collected from macrophages on both surfaces were cultured with gingival fibroblasts seeded on their respective topographies. All experiments were performed in triplicate with three independent experiments.

Results Macrophages seeded on SLA surfaces polarized towards tissue-inflammatory M1 macrophages at early time points. Immunofluorescent staining and RT-PCR analysis demonstrated higher levels of iNOS and gene expression of
\end{abstract}

Yufeng Zhang

zyf@whu.edu.cn

1 The State Key Laboratory Breeding Base of Basic Science of Stomatology (Hubei-MOST) and Key Laboratory of Oral Biomedicine Ministry of Education, School and Hospital of Stomatology, Wuhan University, Wuhan 430079, People's Republic of China

2 Department of Dental Implantology, School and Hospital of Stomatology, Wuhan University, Wuhan 430079, People's Republic of China

3 Department of Periodontology, University of Bern, Bern, Switzerland

4 Department of Periodontology, College of Dental Medicine, Nova Southeastern University, Fort Lauderdale, Florida, USA
IL-1 $\beta$, IL-6, and TNF-alpha on SLA surfaces at 3 days when compared to both tissue culture plastic (TCP) and PT surfaces $(p<0.001)$. Very little differences were found between smooth PT surfaces and TCP. Interestingly, proliferation assay (CCK-8) suggested that conditioned media (CM) from macrophages seeded on SLA surfaces drastically inhibited gingival fibroblast proliferation at 3 and 5 days $(p<0.001)$. Meanwhile, CM from macrophages cultured on SLA surfaces also significantly reduced collagen 1 synthesis on SLA surfaces at 14 days as assessed by immunofluorescent staining $(p<0.001)$.

Conclusion The results from this study demonstrate that the polarization of macrophages towards a pro-inflammatory (M1) phenotype on SLA surfaces may have a negative impact on gingival fibroblast behavior on titanium surfaces. Future strategies to better modulate macrophage polarization should be investigated to support a favorable immune response and encourage tissue integration.

Clinical relevance As SLA surfaces have a potential to shift macrophages towards tissue-inflammatory M1 macrophages, this might be a negative impact for soft tissue healing. Therefore, SLA surfaces should be kept within the bone, as when in contact with soft tissue, they are prone to support a lack of soft tissue integration leading to inflammation.

Keywords Macrophages $\cdot$ M1 and M2 polarization · Immune response $\cdot$ Surface topography $\cdot$ Dental implants

\section{Introduction}

The host-material interface between a biomaterial and a living organism is a complex but crucial interface largely dictating the fate of material tissue integration [1]. At the center of these interactions are immune cells, a group of cells responsible for 
orchestrating various cell types involved in either material biocompatibility or material rejection [1]. Macrophages are key contributors towards the immune response to foreign materials with the ability to promote either a tissue-inflammatory (M1 macrophages) or tissue wound healing microenvironment (M2 macrophages). Despite their importance in tissue homeostasis, their study as it relates to biomaterials has been largely omitted over the years [1].

Dental implants are commonly fabricated utilizing commercially pure titanium, a highly biocompatible material with long-term success rates routinely found over 90\% [2-5]. Despite this, it remains intriguing that to this day, a small percentage of dental implants is lost every year for completely unknown reasons non-related to material failure, and periimplant infections including peri-implant mucositis and periimplantitis are continuously rising [6]. It has been reported that the frequencies of the peri-implant mucositis and periimplantitis have been reported as high as 54 and 28\%, respectively [7]. In order to achieve long-term stability of dental implants, peri-implant soft tissue healing is considered a crucial factor due to its function as a barrier from the oral cavity and their associated microbes [8-10]. A quality soft tissue seal is further necessary to prevent gingival recession or, in the worst case, biomaterial loss [8-10].

Human gingival fibroblasts (HGFs) play a prominent role in soft tissues around dental implants and act as the major cell type responsible for creating a functional seal from the outside mucosa [8-10]. Several studies have noted that the adhesion and function of HGFs on titanium may be further regulated by substratum surface topography [11-23]. While both smooth and rough surfaces have typically been shown to alter fibroblast spreading and contribute to the formation of a continuous collagenous matrix [17], their inter-play with immune cells has not yet been characterized.

To date, studies of immune cells (mainly macrophages) have demonstrated that additional titanium surface roughness tends to promote an increase in M1 pro-inflammatory macrophages by secreting higher levels of M1 pro-inflammatory cytokines including tumor necrosis factor alpha (TNF- $\alpha$ ), interleukin-6 (IL-6), and interleukin-1 $\beta$ (IL-1 $\beta$ ) [24-30]. A hydrophilic moderately rough titanium surface with nanotopography (modSLA) tends to modulate the inflammatory response and promote the polarization of macrophages towards an M2 phenotype both in vitro and in vivo $[31,32]$. While these studies have shown that surface topography influences macrophage polarization and their secretion of proor anti-inflammatory cytokines [28], very little is known concerning how this microenvironment might affect fibroblast behavior and soft tissue connective tissue attachment: a crucial necessity for dental implant longevity and success.

Therefore, the purpose of this study is twofold: first, the effect of titanium surface topography was investigated on macrophage polarization towards either an M1 or M2 phenotype. Thereafter, conditioned media (CM) collected from macrophages seeded on various titanium surfaces was harvested and cultured with HGFs grown on respective smooth and rough titanium surfaces. The effect of CM was investigated on HGF cell adhesion, spreading, proliferation, inflammatory pathway, as well as the ability for HGFs to form a confluent collagen matrix spanning the entire titanium surface.

\section{Materials and methods}

\section{Preparation of PT and SLA titanium discs}

Commercially pure grade 4 titanium was used to fabricate (1) pickled titanium (PT) and (2) Sand-blasted with Large grit particles followed by Acid-etching (SLA) surfaces. Fifteenmillimeter-diameter titanium discs were provided by Straumann AG (Basel, Switzerland) that fit directly into the bottom of 24-well culture plates. Briefly, smooth PT surfaces were prepared using dilute nitric acid to clean the surfaces, followed by washing in reverse osmosis purified water. Roughened SLA topography surfaces were prepared by blasting the titanium with corundum particles, followed by etching with $\mathrm{HCl} / \mathrm{H}_{2} \mathrm{SO}_{4}$.

\section{Cell culture}

The murine-derived macrophage cell line RAW 264.7 (China Center for Type Culture Collection (CCTCC)) and HGFs were used in this study. For macrophage experiments, RAW 264.7 cells were seeded on (1) tissue culture plastic (TCP), (2) PT surfaces, or (3) SLA surfaces in 24-well plates containing Dulbecco's modified Eagle's medium (DMEM) supplemented with $10 \%$ fetal bovine serum (FBS) and 1\% penicillin/ streptomycin (HyClone, Thermo Fisher Scientific Inc) at $37^{\circ} \mathrm{C}$ in a humidified $5 \% \mathrm{CO}_{2}$ atmosphere.

HGFs were obtained from young healthy donors undergoing wisdom tooth extraction, without periodontal diseases as previously described [33]. All volunteers signed an ethical approval and gave full consent in agreement with the IRB committee at the University of Wuhan, China. Collected tissues were washed three times with phosphate-buffered saline (PBS; $150 \mathrm{mM} \mathrm{NaCl}, 20 \mathrm{mM}$ sodium phosphate $\mathrm{pH}$ 7.2) supplemented with $1 \%$ penicillin/streptomycin and cut into small pieces with sterilized surgical scissors. The gingival tissue pieces were then transferred into T25 tissue culture flasks containing minimal DMEM and were allowed to adhere for $2 \mathrm{~h}$ and then replaced by DMEM containing $20 \%$ fetal bovine serum and $1 \%$ penicillin/streptomycin. After 1 week when cells reached confluency, cells were sub-cultured using trypsin and cultured in DMEM with 10\% fetal bovine serum. All cells 
between the third and seventh passage were used for experiments utilizing HGFs.

\section{Collection of conditioned media}

As macrophages cultured on titanium release pro- and antiinflammatory cytokines in the culture media, this could represent a way to target the growth factors that could potentially be released around implant surfaces in vivo creating either a proor an anti-inflammatory microenvironment. Thereafter, gingival fibroblasts were cultured in this conditioned media that could better mimic the in vivo response towards when fibroblasts contact implant surfaces.

RAW 264.7 cells were cultured on TCP, PT, and SLA surfaces in the 24-well plates at a density of $10^{5}$ cells per well. For CM experiments, RAW cells were cultured on the various topographies for 3 days and $\mathrm{CM}$ was then collected and centrifuged at $1500 \mathrm{rpm}$ for $20 \mathrm{~min}$ at $4{ }^{\circ} \mathrm{C}$, and frozen at $-80^{\circ} \mathrm{C}$ until experimental seeding.

\section{Adhesion and proliferation assay of RAW 264.7 cells cultured on titanium surfaces}

RAW 264.7 cells were seeded on TCP, PT, and SLA surfaces in 24-well plates at a density of $2 \times 10^{4}$ cells per well. At time points 2, 4, and $8 \mathrm{~h}$ for cell adhesion and 1 and 3 days for cell proliferation, a Cell Counting Kit-8 (Dojindo, Japan) was used to determine cell numbers as previously described [34]. After incubation for $1.5 \mathrm{~h}$, complete culture medium with $10 \%$ CCK- 8 was removed and washed three times with PBS, then replaced with a culture medium. The absorbance was measured using a microplate reader scanning at $450 \mathrm{~nm}$ (PowerWave XS2, BioTek, Winooski, VT, USA). Samples were performed in triplicate with three independent experiments performed.

\section{Inflammatory gene expression of RAW 264.7 cells cultured on titanium surfaces}

RAW 264.7 were cultured on the TCP, PT, and SLA surfaces in 24-well plates at a density of $10^{5}$ cells per well for real-time PCR experiments. Total RNA was extracted from RAW 264.7 after 3 days using AxyPrep ${ }^{\mathrm{TM}}$ Multisource Total RNA Miniprep Kit (Axygen, Union City, CA USA) according to the manufacturer's instruction. The RNA concentration was measured using a NanoDrop 2000 UV-Vis Spectrophotometer as previously described [35]. Thereafter, $1 \mu \mathrm{g}$ of total RNA was used for complementary DNA (cDNA) synthesis using a Transcriptor First Strand cDNA Synthesis Kit (Roche, Basel, Switzerland).

RT-PCR primers including inflammatory-related cytokines such as TNF- $\alpha$, IL-6, IL-1 $\beta$, and GAPDH genes were utilized in this study (listed in Table 1). RT-PCR was performed using $25-\mu$ final reaction volume of QuantiFast ${ }^{\mathrm{TM}}$ SYBR Green
PCR Kit (QIAGEN, Venlo, Holland), and the target gene expression was assayed on a CFX Connect ${ }^{\mathrm{TM}}$ Real-Time PCR Detection System. The delta-delta Ct method was used to calculate gene expression levels relative to housekeeping gene GAPDH and normalized to control cells (TCP group). Each sample contained pooled messenger RNA (mRNA) collected from three titanium surfaces, and all samples were log transformed. The experiments were performed in triplicate with three independent experiments.

\section{M1 and M2 surface marker changes of RAW 264.7 cells cultured on titanium surfaces}

Immunofluorescence assay was used to reveal the expression of $\mathrm{M} 1$ and $\mathrm{M} 2$ cell surface markers inducible nitric oxide synthase (iNOS) and CD206, respectively. Briefly, RAW 264.7 were cultured in 12 -well plates at a density of $2 \times 10^{4}$ cells per well. After 3 days, the cells were rinsed three times with PBS and fixed in $4 \%$ formaldehyde for $15 \mathrm{~min}$. Cells were then incubated with primary antibody iNOS $(1: 100$, Santa Cruz, USA) and CD206 (1:100, Santa Cruz, USA) (both $\operatorname{IgG}_{1} \mathrm{FAB}$ fragment, kappa light chain), respectively, diluted in PBS containing $2 \%$ bovine serum albumin (BSA, Roche) for $1 \mathrm{~h}$, followed by incubating with the secondary antibody FITC-conjugated goat anti-rabbit (1:200, Invitrogen) for $1 \mathrm{~h}$. Thereafter, 4',6-diamidino-2-phenylindole (DAPI) was added for visualizing the cell nuclei as previously described [36]. Images were taken on an Olympus DP71 fluorescence microscope (Olympus Co, Japan).

\section{Adhesion assay of human gingival fibroblasts cultured on titanium surfaces with conditioned media from macrophages}

HGFs were seeded at a density of $2.5 \times 10^{4}$ cells per well on (1) TCP, (2) PT, and (3) SLA surfaces in 12-well plates with or without $\mathrm{CM}$ and cultured for 2,4 , and $8 \mathrm{~h}$ for the adhesion assay. For counting the cell number, DAPI was applied to visualize the nuclei as previously described [36]. At each time point, cells were rinsed with PBS three times to remove the unattached cells and fixed in $4 \%$ formaldehyde for $10 \mathrm{~min}$ followed by staining with DAPI. Images were taken on an Olympus DP71 fluorescence microscope (Olympus Co, Japan). Ten fields of view were taken per sample at random and nuclei were counted using the ImageJ software (Maryland, USA).

\section{Human gingival fibroblast morphology cultured on titanium surfaces with conditioned media from macrophages}

HGFs were seeded on TCP, PT, and SLA in 12-well plates at a density of $2.5 \times 10^{4}$ cells per well either with or without $\mathrm{CM}$. 
Table 1 Primer pairs used in the qRT-PCR

\begin{tabular}{llll}
\hline Genes & $\begin{array}{l}\text { Forward } \\
\left(5^{\prime}-3^{\prime}\right)\end{array}$ & $\begin{array}{l}\text { Reverse } \\
\left(5^{\prime}-3^{\prime}\right)\end{array}$ & $\begin{array}{l}\text { Product size } \\
(\mathrm{bp})\end{array}$ \\
\hline TNF- $\alpha$ & CTGAACTTCGGGGTGATCGG & GGCTTGTCACTCGAATTTTGAGA & 122 \\
IL-6 & ATAGTCCTTCCTACCCCAATTCC & GATGAATTGGATGGTCTTGGTCC & 93 \\
IL-1ra & CTCCAGCTGGAGGAAGTTAAC & CTGACTCAAAGCTGGTGGTG & 109 \\
IL-1 $\beta$ & TGGAGAGTGTGGATCCCAAG & GGTGCTGATGTACCAGTTGG & 128 \\
IL-10 & GAGAAGCATGGCCCAGAAATC & GAGAAATCGATGACAGCGCC & 96 \\
IL-4 & ACAGGAGAAGGGACGCCAT & GAAGCCCTACAGACGAGCTCA & 95 \\
COL1A1 & TCTAGACATGTTCAGCTTTGTGGAC & TCTGTACGCAGGTGATTGGTG & 134 \\
FN1 & ACCTACGGATGACTCGTGCTTTGA & CAAAGCCTAAGCACTGGCACAACA & 116 \\
VCL & TCAGATGAGGTGACTCGGTTGG & TTATGGTTGGGATTCGCTCACA & 103 \\
GAPDH & GCACCGTCAAGGCTGAGAAC & TGGTGAAGACGCCAGTGGA & 138 \\
\hline
\end{tabular}

At time point $2,4,8$, and $24 \mathrm{~h}$, cells were fixed in $4 \%$ formaldehyde for $10 \mathrm{~min}$ followed by rinsing with PBS. Five micrograms per milliliter phalloidin-FITC (Sigma-Aldrich) was used to show the morphology of cells as previously described [37]. Then DAPI was used to visualize the nuclei. For each group, images were taken on an Olympus DP71 fluorescence microscope (Olympus Co, Japan) and compared for morphological differences. The average surface area of cells was measured using the ImageJ software (Maryland, USA).

\section{Proliferation assay of human gingival fibroblasts cultured on titanium surfaces with conditioned media from macrophages}

HGFs were seeded on TCP, PT, and SLA surfaces in 24-well plates at a density of $1 \times 10^{4}$ cells per well either with or without CM. At time point 1, 3, and 5 days, Cell Counting Kit-8 (Dojindo, Japan) was used to investigate cell proliferation as previously described [34]. The experiments were performed in triplicate with three independent experiments performed.

\section{Adhesion-related gene expression of human gingival fibroblasts cultured on titanium surfaces with conditioned media from macrophages}

HGFs were cultured on TCP, PT, and SLA surfaces in 24-well plates at a density of $5 \times 10^{4}$ cells per well either with or without CM for real-time PCR experiments. After 3 days of culture, total RNA was isolated from HGFs for detection of the expression of collagen 1 (COL1), fibronectin (FN1), and vinculin (VCL) genes using RT-PCR as previously described. GAPDH was used as a housekeeping gene, and experiments were performed in triplicate with three independent experiments.

\section{Collagen immunofluorescence staining assay}

HGFs were seeded at a density of $1 \times 10^{4}$ cells per well in 12 well plate either with or without CM. The culture medium was changed every 2 days. At 14 days, the cells were rinsed with PBS three times and fixed in $4 \%$ formaldehyde for $15 \mathrm{~min}$. Then, $0.5 \%$ Triton X-100 (Merck, Germany) was used to permealize cells. Subsequently, cells were incubated with polyclonal rabbit to collagen type 1 (1:100, Boster Biological Technology Ltd., Wuhan, China) diluted in PBS containing $2 \%$ bovine serum albumin (BSA, Roche) for $1 \mathrm{~h}$, followed by incubation with FITC-conjugated goat anti-rabbit (1:200, Invitrogen) diluted in PBS containing 2\% BSA for $1 \mathrm{~h}$. Finally, cells were reacted with DAPI for $3 \mathrm{~min}$. After each step, the cells were washed with PBS three times. Images were taken on an Olympus DP71 fluorescence microscope (Olympus Co, Japan).

\section{Statistical analysis}

Statistical analysis was performed by one-way ANOVA for real-time PCR analysis and quantification of collagen 1 staining analysis. Two-way ANOVA was utilized for the cell adhesion assay, cell proliferation assay, and average surface area assay followed by Tukey post hoc test, using GraphPad Software v.6 (GraphPad Software, La Jolla, CA, USA). Statistical significance was set at $p<0.05$. All data are expressed as the mean $\pm \mathrm{SE}$.

\section{Results}

\section{The effect of titanium on macrophage adhesion and proliferation}

The effect of surface topography was first investigated on macrophage adhesion and proliferation (Fig. 1). It was 
observed that all surface topographies demonstrated high attachment, and no significant differences were observed between all groups at 2,4 , or $8 \mathrm{~h}$ post-seeding (Fig. 1a). Analysis of cell proliferation demonstrated that at 1 day post-seeding; no difference was observed between control TCP, PT, or SLA surfaces (Fig. 1b). By 3 days, however, macrophages seeded on PT and SLA surfaces showed significantly higher cell numbers when compared to control TCP ( $p<0.001$ ) (Fig. 1b). SLA demonstrated the significantly highest cell numbers at 3 days when compared to control TCP and PT surfaces $(p<0.001)$ (Fig. 1b).

\section{Inflammatory gene expression of RAW 264.7 cells cultured on titanium surfaces}

Thereafter, gene expression of M1 and M2 macrophage markers including IL- $1 \beta$, TNF- $\alpha$, IL-6, and IL-1ra expression was investigated at 3 days (Fig. 2). It was found that RAW cells seeded on rough SLA surfaces increased the expression of mRNA levels of pro-inflammatory markers including IL$1 \beta$, IL-6, TNF-alpha, and IL-1ra when compared to both TCP and PT surfaces $(p<0.001)$ (Fig. $2 \mathrm{a}-\mathrm{d})$. Neither of the tissueresolving interleukins including IL-4 nor IL-10 demonstrated any changes between all investigated groups (Fig. 2e, f).

\section{M1 and M2 surface markers of RAW cells cultured on titanium surfaces}

M1 and M2 macrophage cell surface marker iNOS (M1) and CD206 (M2) were then investigated utilizing immunofluorescence at 3 days (Fig. 3). It was observed that CD206 expression on both PT and SLA surfaces was visibly more apparent than on TCP surfaces. No differences could be observed between PT and SLA surfaces (Fig. 3). Interestingly, the expression of iNOS, an M1 pro-inflammatory macrophage marker, was seen visibly more highly stained in RAW cells seeded on SLA surfaces when compared to TCP and PT surfaces (Fig. 3 ). This finding confirms previous mRNA data indicating that

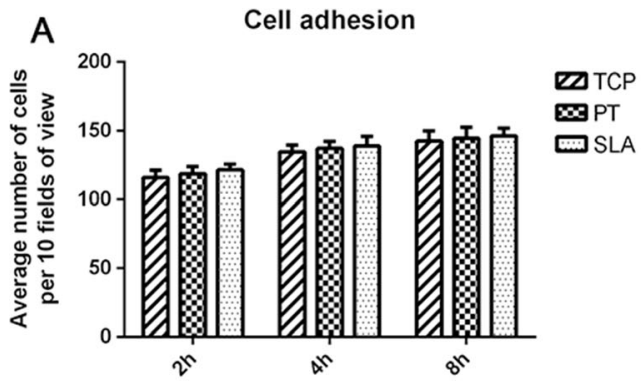

Fig. 1 Effect of surface topography on RAW264.7 cell adhesion and proliferation for (1) tissue culture plastic (TCP), (2) PT, and (3) SLA samples. a Cell adhesion at 2, 4, and $8 \mathrm{~h}$. b Cell proliferation at 1 and 3
SLA surfaces promote macrophage polarization towards the M1 pro-inflammatory macrophage.

\section{Human gingival fibroblast attachment to titanium surfaces with conditioned media from macrophages}

Thereafter, a series of experiments investigating the effects of CM from macrophages on HGF behavior was performed. First, HGFs attached to all surfaces with or without CM at 2, 4 , and $8 \mathrm{~h}$. At early time points $(2 \mathrm{~h})$, it was observed that significantly less cells were found on TCP and PT surfaces cultured with CM $(p<0.001)$ (Fig. 4b). Furthermore, control SLA surfaces demonstrated significantly higher cell attachment at $2 \mathrm{~h}$ when compared to control TCP and control PT surfaces both with and without conditioned media $(p<0.001)$ (Fig. 4b). By 4 and 8 h, a similar number of HGFs was observed on all surfaces irrespective of surface topography or addition of CM (Fig. 4b).

The morphology of HGFs was then investigated in the presence or absence of CM at 2, 4, 8, and $24 \mathrm{~h}$ (Fig. 4a). It was first found that after $2 \mathrm{~h}$, cells had a round appearance with very little spreading occurring at $2 \mathrm{~h}$, whereas by $24 \mathrm{~h}$, cell spreading on all three surfaces became apparent. Interestingly, the addition of CM to culture media tended to inhibit the spreading of HGFs, most notably on SLA surfaces (Fig. 4a). Analysis of cell surface area demonstrated that CM significantly reduced cell surface area on both PT and SLA surfaces at 8 and $24 \mathrm{~h}$ when compared to their respective controls $(p<0.001)$ (Fig. 4c).

\section{Human gingival fibroblast proliferation}

At 1 day post-seeding, no significant differences were observed in HGF numbers between all groups, regardless of surface topography or the presence of CM (Fig. 4d). At both 3 and 5 days, however, it was found that cells cultured on control SLA demonstrated significantly lower cell numbers in comparison to control TCP and control PT surfaces $(p<0.001)$. When CM was added to culture media,

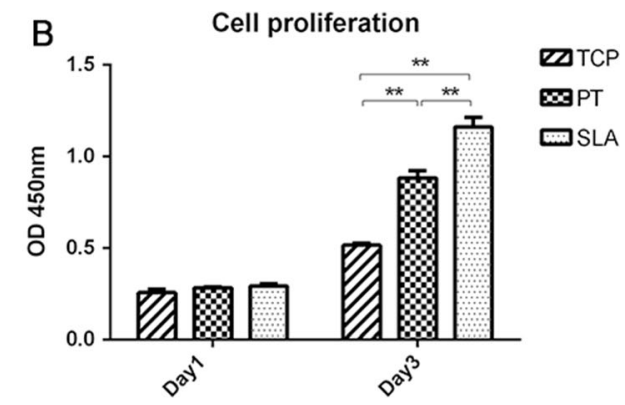

days. Data are means $\pm \mathrm{SE}$. Double asterisks denote significant difference between titanium surface and TCP surfaces, $p<0.001$ 

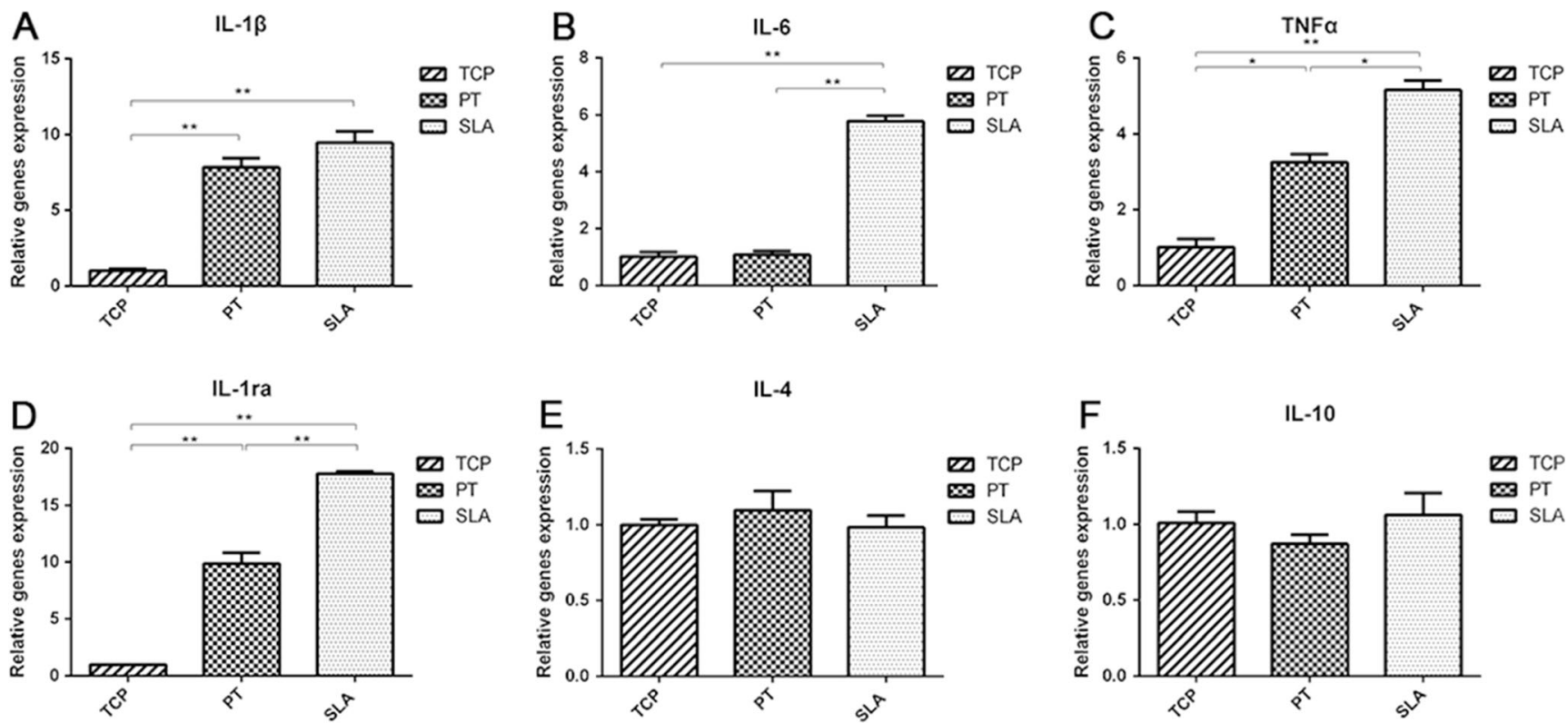

Fig. 2 Relative mRNA expression of inflammation related genes: IL-1 $\beta$, IL-6, TNF- $\alpha$, IL-1ra, IL-4, and IL-10 by RAW264.7 seeded on TCP, PT, and SLA at $72 \mathrm{~h}$. GAPDH was used as housekeeping genes (Double

asterisks denote significant difference between titanium surface and TCP surfaces, $p<0.001$. Single asterisk asterisk denotes differences between titanium surface and TCP surfaces $p<0.05$

significantly lower cell proliferation was observed at both 3 and 5 days on all surfaces $(p<0.001)$ (Fig. 4d). Notably, the addition to CM from SLA surfaces inhibited HGF cell proliferation to a much larger degree between 1, 3, and 5 days $(p<0.001)$ (Fig. 4d).

\section{Human gingival fibroblast expression of collagen 1, fibronectin, and vinculin}

The effect of CM was then investigated on gene expression of COL1, FN1, and VCL at 3 days. It was found that CM significantly downregulated the expression of COL1 on all surfaces when compared to their respective controls $(p<0.001)$ (Fig. 5a). Once again, SLA surfaces had the most pronounced and detrimental effect whereby addition of CM significantly decreased COL1 mRNA levels over tenfold $(p<0.001)$ (Fig. $5 a)$. mRNA levels of cell adhesion molecules including FN1 and VCL did not significantly change between control and $\mathrm{CM}$ groups on all tested titanium surface topographies (Fig. $5 b, c)$. On TCP, FN1 and VCL gene expression was significantly higher with CM (Fig. 5b, c).

\section{Collagen type 1 staining}

Collagen type 1 immunofluorescent staining was then utilized to visualize the extracellular matrix deposition of
Fig. 3 Immunofluorescence images showing M1 and M2 surface markers iNOS and CD206 of RAW cells, respectively, on TCP, PT, and SLA surface at 3 days postseeding. Higher staining intensity was qualitatively observed for iNOS on SLA surfaces when compared to TCP and PT surfaces, respectively $($ Bar $=$ $100.00 \mu \mathrm{m})$
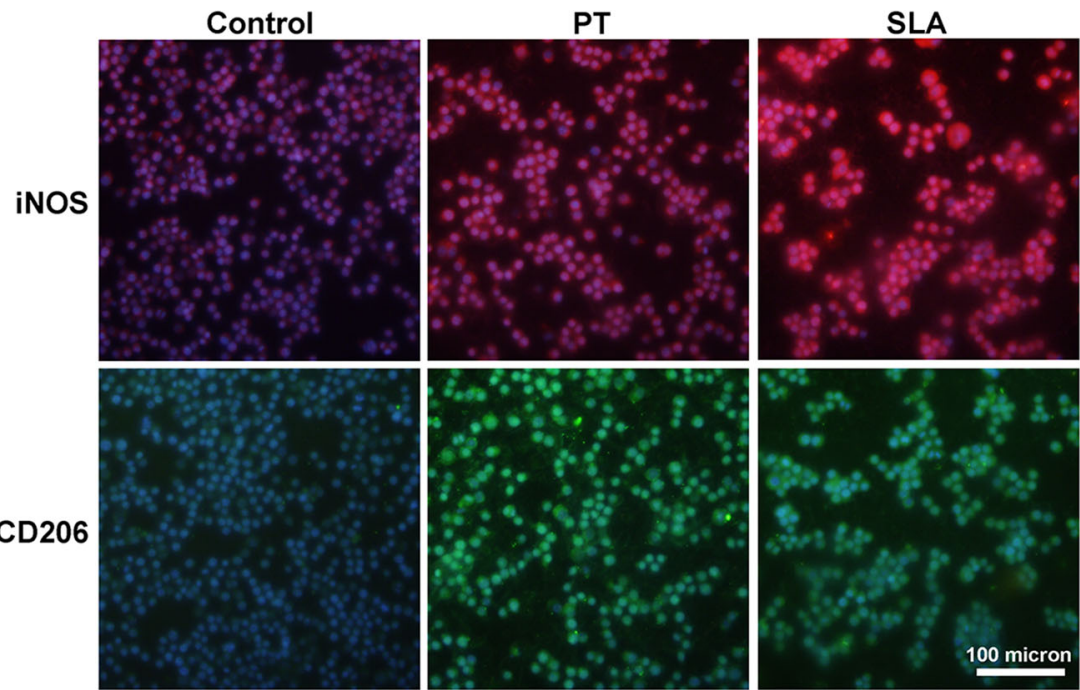
A

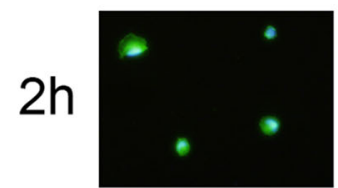

$4 h$

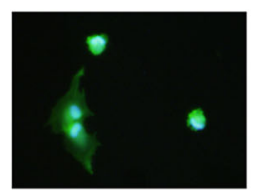

$8 h$

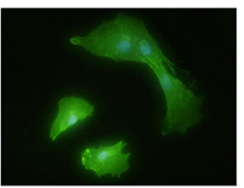

$24 h$

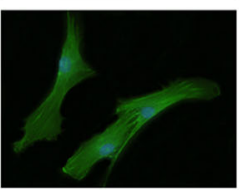

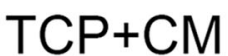
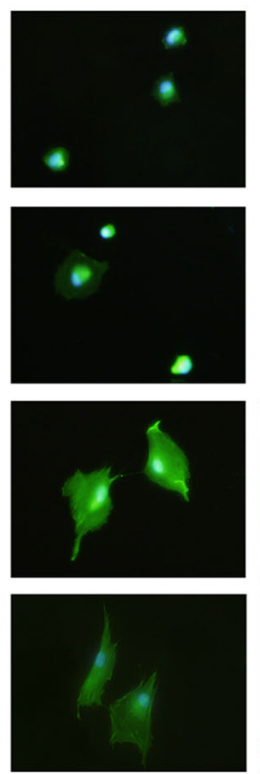
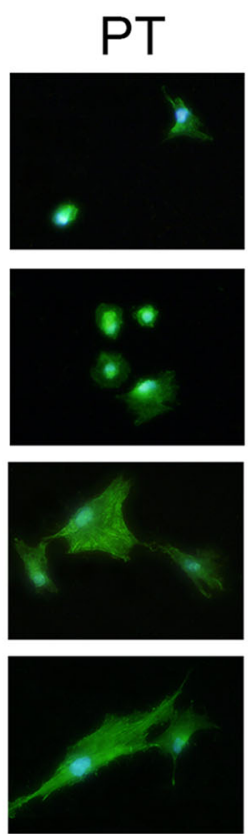
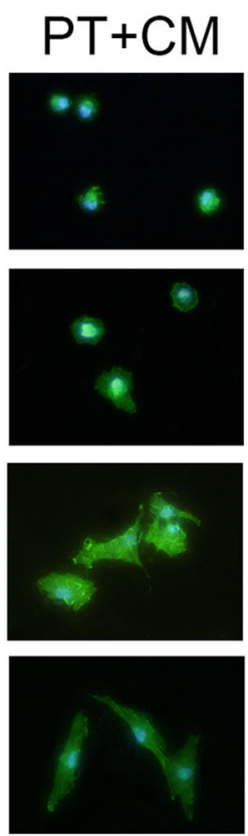

SLA
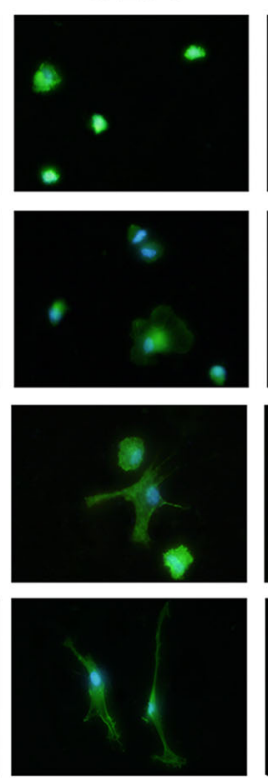

$\mathrm{SLA}+\mathrm{CM}$
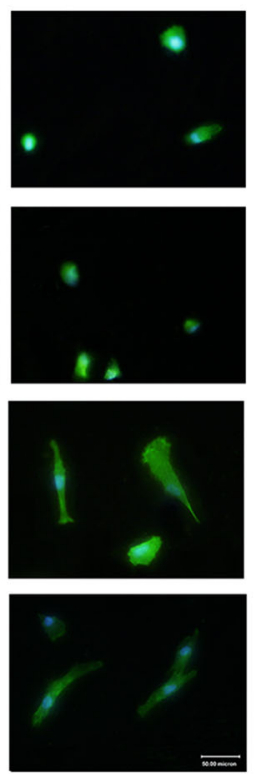

B

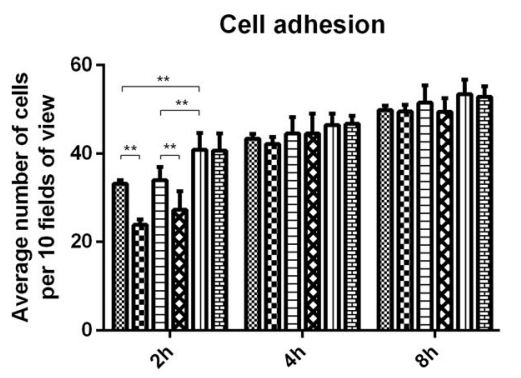

C

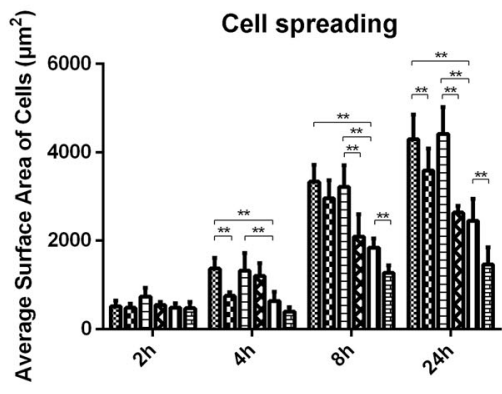

Cell spreading
D
D

(1)

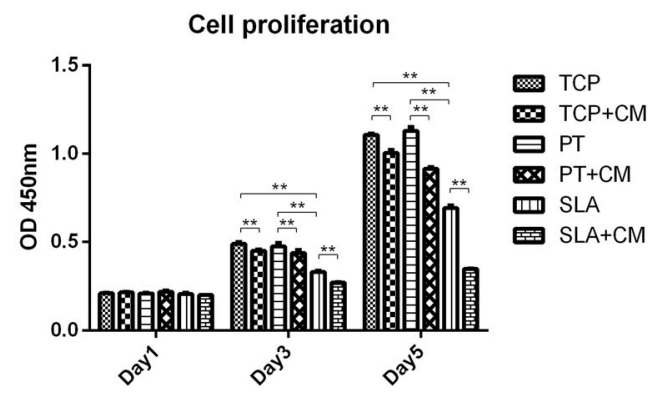

Fig. 4 Effects of surface topography and conditioned media (CM) on human gingival fibroblast cell adhesion and proliferation on (a) TCP control, (b) TCP + CM, (c) PT control, (d) PT + CM, (e) SLA control, and (f) SLA + CM. a Spreading of human gingival fibroblasts cultured with and without conditioned media (CM) demonstrated qualitatively less cell spreading for cells cultured with $\mathrm{CM}$ when compared to their respective controls. Cells were stained for F-actin (green) and nuclei (blue). b Effects of surface topography and conditioned medium (CM) on human gingival fibroblast cell adhesion at 2, 4, and $8 \mathrm{~h}$ post-seeding. $\mathbf{c}$ Average surface planar area of cells. It was found that cells cultured with

human gingival fibroblasts onto titanium surfaces (Fig. 6). At 2 weeks post-seeding, COL1 expression on PT surfaces was visibly more apparent than on TCP and SLA surfaces irrespective of CM (Fig. 6a). No differences could be observed between TCP and SLA surfaces with respect to staining intensity of COL1 (Fig. 6a). Interestingly, COL1 staining of cells cultured on SLA surface with CM was markedly and significantly decreased when compared to SLA controls as well as to all other surfaces (Fig. 6a). It was further observed that collagen staining was in the SLA + CM group revealed staining mainly concentrated within the cytoplasm of HGFs seeded on SLA + CM surfaces with little to no extracellular
CM showed significantly less cell spreading when compared to respective control cells. d Cell proliferation investigation revealed that cells cultured on SLA showed significantly lower cell numbers when compared to TCP and PT surfaces. Furthermore, cells stimulated with respective CM significantly inhibited cell numbers at 3 and 5 days post-seeding; most notably for SLA surfaces. Data are means \pm SE. (Double asterisks denote significant difference between titanium surface and TCP surfaces, $p<0.001$. Single asterisk denotes differences between titanium surface and TCP surfaces $p<0.05$. Data are means \pm SE. $($ Bar $=50.00 \mu \mathrm{m})$

matrix deposition (Fig. 6a). Quantification of staining area confirmed these findings and the significant loss of collagen deposition when CM from macrophages on SLA was added $(p<0.001)$ (Fig. 6b).

\section{Discussion}

The role of immune cells around biomaterials has been a topic of study both in vitro and in vivo over several decades [38]. While research from over 20 years ago made it clear that cells from the monocytic lineage were one of the first to come in contact with various biomaterials [39], it is interesting to point 
A
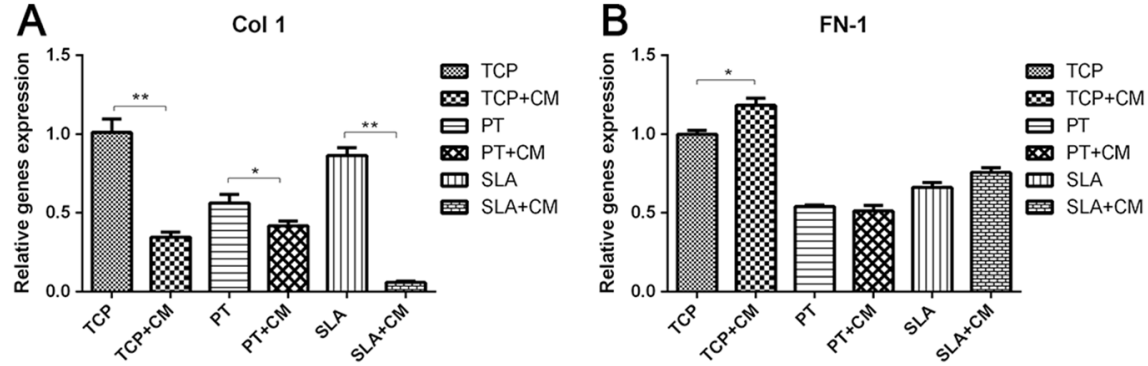

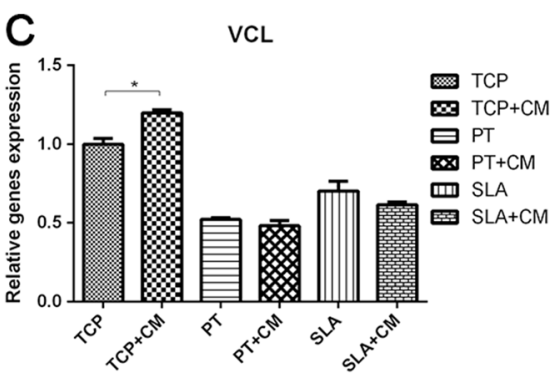

Fig. 5 Relative mRNA expression of (a) collagen 1 (COL1), (b) fibronectin (FN1), and (c) vinculin (VCL) of human gingival fibroblasts seeded on TCP, PT, and SLA at $24 \mathrm{~h}$ with and without conditioned media (CM). Most notably, CM significantly downregulated mRNA levels of
COL1 on all surfaces; most notably on SLA surfaces. Data are means $\pm \mathrm{SE}$ (single asterisk denotes differences between titanium surface and TCP surfaces $p<0.05$; double asterisks denote significant difference between titanium surface and TCP surfaces, $p<0.001$ ) out that despite widespread study of immune cell interactions with various medical biomaterials [38], their role in implant dentistry has largely been left unstudied [1]. In fact, a recent systematic review gathering all in vitro studies relating to cells and implant surfaces found that approximately $90 \%$ of all published studies focused primarily on connective tissue behavior (either gingival fibroblasts or osteoblasts) while only a small percentage (roughly 10\%) was dedicated to immune cells including monocytes, macrophages, osteoclasts, leukocytes, and multinucleated giant cells [40]. This finding grossly demonstrates the lack of study with immune modulation of implant surfaces despite the fact that tissue integration is routinely found preceded by macrophage accumulation [41]

To date, much research has investigated the effect of surface topography on cell behavior $[12,13]$. It has been shown that gingival fibroblasts tend to favor microroughened surfaces or

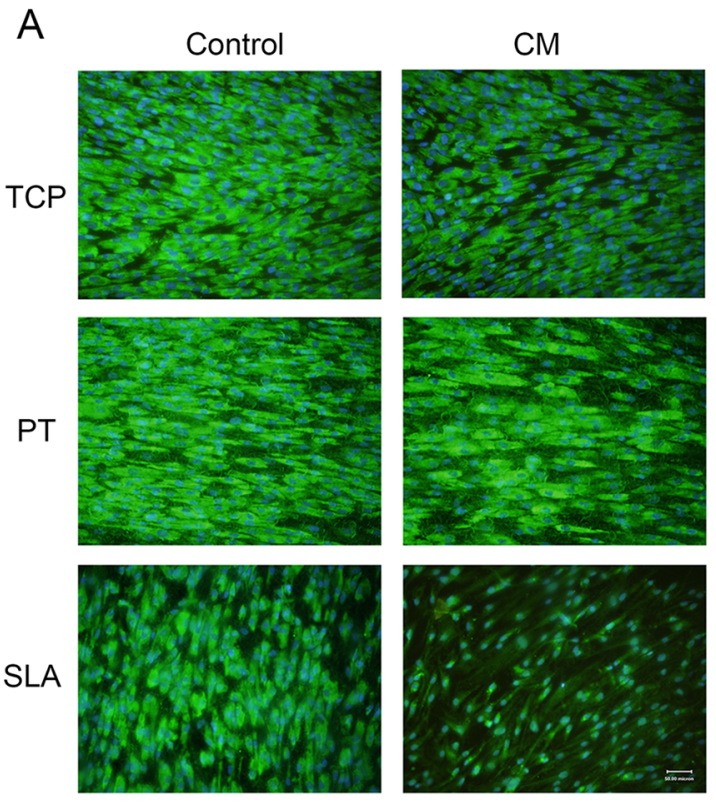

Fig. 6 Immunofluorescence staining of collagen type 1 for human gingival fibroblasts seeded on pickled titanium (PT) and Sand-blasted with Large grit particles followed by Acid-etching (SLA) surfaces with and without conditioned media (CM) at 2 weeks post-seeding. While PT surfaces demonstrated slightly higher expression of collagen type 1 when compared to all other surface, the major finding was that the addition of $\mathrm{CM}$ grooved surfaces capable of "guiding" fibroblast behavior [12, 13]. In an attempt to improve the soft tissue seal of transmucosal titanium implants, recent investigations have shown that certain nanotopographies may be utilized to guide in a predetermined way the orientation of aligned collagen fibers on titanium surfaces both in vitro and in vivo [14-16]. Much of this work originated from basic cell studies conducted in the 1990s demonstrating that the order in which fibroblasts encounter substratum topography and extracellular matrix could influence matrix and cell orientation [17-22].

Despite the finding that surface microroughness may improve gingival cell behavior, one of the drawbacks of increased surface roughness is the pro-inflammatory response of macrophages in contact with roughened surfaces. While one study has shown that surface roughness increases regenerative molecules such as transforming growth factor beta 1 as well as bone morphogenetic

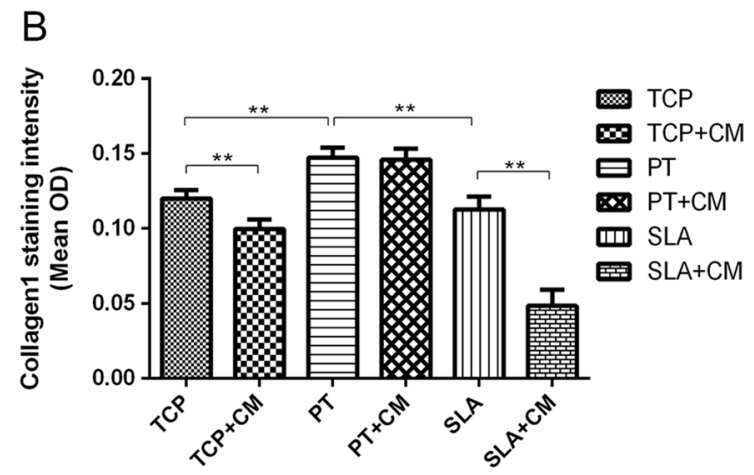

from macrophages significantly decreased collagen type 1 expression notably on SLA surfaces. a The merged fluorescent images of collagen type 1 staining (green) with DAPI staining (blue). Bar $=50.00 \mu \mathrm{m}$. b Quantified values of collagen type 1 staining in comparison to the control TCP sample. Data are means \pm SE. Double asterisks denote significant difference between titanium surface and TCP surfaces, $p<0.001$ 
protein 2 [42], the majority of in vitro work to date has demonstrated that roughness tends to increase pro-inflammatory cytokine expression including IL-1beta, IL-6, TNF-alpha, and CCL2 [25-30]. Furthermore, roughened surfaces have also been shown to increase monocyte chemoattractant protein- 1 and macrophage inflammatory protein-1alpha further contributing to subsequent recruitment of macrophages and local inflammation [28].

While previous studies show that surface topography has both an influence on fibroblast behavior as well as macrophage M1 polarization, to date, no study has investigated their interrelationship and inter-communication between these two cell types. Therefore, the purpose of this study was to focus more directly on how immune cells may potentially modulate fibroblast behavior cultured on biomaterial surfaces. Herein, we confirm previous findings that surface roughness significantly increases pro-inflammatory M1 macrophage phenotypes in the early period; however, we show how collected CM from macrophages cultured on roughened SLA surfaces clearly and significantly affect gingival fibroblast proliferation and decisively inhibit their ability to form a collagen layer over the implant surface. This striking and novel data demonstrates that $\mathrm{CM}$ from macrophages seeded on SLA surfaces significantly inhibited fibroblast proliferation over twofold, and decreased collagen mRNA levels over tenfold (Figs. 5 and 6). We therefore show convincingly for the first time that in fact, immune cells play a much more prominent role in tissue integration than does surface topography, and largely dictate the fate of biomaterial tissue integration. Future strategies employed to better control macrophage polarization in response to various biomaterials are thus warranted and necessary.

Another clinical aspect largely discouraging the use of roughened surfaces at the transmucosal portion of the dental implant has been the severe effects of increased bacterial adherence and colonization onto rougher surfaces [43-45]. Therefore, and for this reason, numerous commercially available dental implants have employed more smooth transmucosal portions of the implant/abutment interface spanning gingival tissues in order to prevent peri-implant disease [46,47]. That being said, Refai et al. further showed that macrophages cultured with lipopolysaccharide (LPS), a bacterial by-product commonly produced by gramnegative bacteria, synergistically increased the release of proinflammatory cytokines produced by macrophages within $24 \mathrm{~h}$ [28]. Therefore, new strategies must be employed to counteract some of these damaging microenvironments, especially with the exponentially increasing rate of peri-implantitis suggested to be in the range of $11-47 \%$ for all implants placed after 10 years [48].

Recently, a prominent group in Sweden working with dental implants for over 30 years has begun investigating the "foreign body reaction" around dental implants [49, 50]. While their key research topic relates to how cells derived from the monocytic lineage are implicated in the long-term equilibrium of biomaterials via the formation of giant foreign body cells (GFBCs) [49-51], results contained within the present study further demonstrate with convincing evidence that these cells are in fact responsible for early biomaterial integration and control cell behavior much more prominently than does surface topography. Therefore, prominent research is absolutely necessary and various biomaterial testing should focus on the immune cell/biomaterial relationship.

Recently, several studies have demonstrated that the wettability of titanium surfaces may be an important factor contributing to the speed of osseointegration by demonstrating that modified SLA (modSLA) surfaces decreased TNF-alpha, IL1-beta, and IL-6 expression when compared to control hydrophobic SLA surfaces and tended to promote the polarization of macrophages to M2 macrophages in both in vitro and in vivo [25-27, 31, 32]. Furthermore, some in vivo studies have investigated that shifts in macrophage polarization at the tissueimplant interface from a pro-inflammatory (M1) to an antiinflammatory (M2) phenotype via various modifications to implant surfaces may improve implant integration [52-55]. Therefore, it remains of interest to further investigate various modifications to implant surfaces and their effect on macrophage polarization. Furthermore, it would be ideal to further optimize the current in vitro culture system by utilizing human primary macrophages as opposed to a murine cell line. It is known that macrophage species has an influence of cytokine secretion, and further investigation on this topic may provide further valuable cues. While our group has shown in the present study the importance of macrophages on gingival cell behavior on titanium surfaces, it remains to be investigated how macrophages dictate the cell fate of osteoblasts seeded on titanium surfaces with varying topographies or diverse implant material compositions including titanium alloys or zirconia implants. Furthermore, since tissue integration of dental implants seems to be modulated at least in part by immune cells, such as macrophages, it remains to be investigated the potential genes/ molecular mechanisms driving this cross talk between macrophages and fibroblasts. Future research is therefore needed.

\section{Conclusion}

In summary, the present study shows that the polarization of macrophages to a pro-inflammatory (M1) phenotype may have a negative impact on gingival fibroblast behavior on titanium surfaces. Roughened implant surfaces caused an increase in the release of pro-inflammatory cytokines in the early period that significantly inhibited the ability for human gingival fibroblasts to proliferate on their respective surfaces, whereas PT surfaces had a much less pronounced effect. Furthermore, macrophage CM from SLA surfaces noticeably inhibited the ability for HGFs to lay a functional collagen matrix across the SLA surface, demonstrating the substantial role of macrophages/immune cells on biomaterial integration. Future study investigating macrophage polarization to biomaterial surfaces and more importantly their inter-play with 
various regenerative cell types is necessary to further improve biomaterial compatibility.

\section{Compliance with ethical standards}

Conflict of interest The authors declare that they have no conflict of interest.

Funding The study was supported by the National Natural Science Foundation of China (81570954 and 81271108). This work was further supported by the International Team for Implantology (ITI), grant number 1154_2016.

Ethical approval This article does not contain any studies with human participants or animals performed by any of the authors. Human gingival fibroblasts were obtained from healthy volunteers that signed an ethical approval and gave full consent.

Informed consent All volunteers whose human gingival fibroblast cells were isolated signed an ethical approval and gave full consent in agreement with the IRB committee at the University of Wuhan, China.

\section{References}

1. Miron RJ, Bosshardt DD (2015) OsteoMacs: key players around bone biomaterials. Biomaterials

2. Romeo E, Lops D, Margutti E, Ghisolfi M, Chiapasco M, Vogel G (2003) Long-term survival and success of oral implants in the treatment of full and partial arches: a 7-year prospective study with the ITI dental implant system. Int J Oral Maxillofac Implants 19(2):247-259

3. Karoussis IK, Salvi GE, Heitz-Mayfield LJA, Brägger U, Hämmerle CHF, Lang NP (2003) Long-term implant prognosis in patients with and without a history of chronic periodontitis: a 10year prospective cohort study of the ITI® Dental Implant System. Clin Oral Implants Res 14(3):329-339

4. Eckert SE, Choi Y-G, Sánchez AR, Koka S (2006) Comparison of dental implant systems: quality of clinical evidence and prediction of 5-year survival. J Prosthet Dent 95(2): 170

5. Buser D, Janner SF, Wittneben JG, Bragger U, Ramseier CA, Salvi GE (2012) 10-year survival and success rates of 511 titanium implants with a sandblasted and acid-etched surface: a retrospective study in 303 partially edentulous patients. Clin Implant Dent Relat Res 14(6):839-851. doi:10.1111/j.1708-8208.2012.00456.x

6. Kronström M, Svensson B, Erickson E, Houston L, Braham P, Persson GR (2000) Humoral immunity host factors in subjects with failing or successful titanium dental implants. J Clin Periodontol 27(12):875-882

7. Gurgel BC, Montenegro SC, Dantas PM, Pascoal AL, Lima KC, Calderon PD (2016) Frequency of peri-implant diseases and associated factors. Clin Oral Implants Res. doi:10.1111/clr.12944

8. Wang Y, Zhang Y, Miron RJ (2015) Health, maintenance, and recovery of soft tissues around implants. Clin Implant Dent Relat Res. doi:10.1111/cid.12343

9. Sculean A, Gruber R, Bosshardt DD (2014) Soft tissue wound healing around teeth and dental implants. J Clin Periodontol 41(Suppl 15):S6-22. doi:10.1111/jcpe.12206

10. Chang YY, Lai CH, Hsu JT, Tang CH, Liao WC, Huang HL (2012) Antibacterial properties and human gingival fibroblast cell compatibility of $\mathrm{TiO}_{2} / \mathrm{Ag}$ compound coatings and $\mathrm{ZnO}$ films on titaniumbased material. Clin Oral Investig 16:95-100. doi:10.1007/s00784010-0504-9
11. Lee H-J, Lee J, Lee J-T, Hong J-S, Lim B-S, Park H-J, Kim Y-K, Kim T-I (2015) Microgrooves on titanium surface affect periimplant cell adhesion and soft tissue sealing; an in vitro and in vivo study. J Periodontal Implant Sci 45(3):120. doi:10.5051/ jpis.2015.45.3.120

12. Mendonça G, Mendonça DBS, Aragao FJL, Cooper LF (2008) Advancing dental implant surface technology-from micron- to nanotopography. Biomaterials 29(28):3822-3835

13. Le Guéhennec L, Soueidan A, Layrolle P, Amouriq Y (2007) Surface treatments of titanium dental implants for rapid osseointegration. Dent Mater 23(7):844-854

14. Kato E, Sakurai K, Yamada M (2015) Periodontal-like gingival connective tissue attachment on titanium surface with nanoordered spikes and pores created by alkali-heat treatment. Dental Mater : Off Pub Acad Dental Mater 31(5):e116-e130. doi:10.1016/ j.dental.2015.01.014

15. Kearns VR, Williams RL, Mirvakily F, Doherty PJ, Martin N (2013) Guided gingival fibroblast attachment to titanium surfaces: an in vitro study. J Clin Periodontol 40(1):99-108. doi:10.1111/ jcpe. 12025

16. Lee HJ, Lee J, Lee JT, Hong JS, Lim BS, Park HJ, Kim YK, Kim TI (2015) Microgrooves on titanium surface affect peri-implant cell adhesion and soft tissue sealing; an in vitro and in vivo study. J Periodontal Implant Sci 45(3):120-126. doi:10.5051/jpis.2015.45.3.120

17. Chou L, Firth JD, Uitto VJ, Brunette DM (1995) Substratum surface topography alters cell shape and regulates fibronectin mRNA level, mRNA stability, secretion and assembly in human fibroblasts. J Cell Sci 108(Pt 4):1563-1573

18. Chou L, Firth JD, Uitto VJ, Brunette DM (1998) Effects of titanium substratum and grooved surface topography on metalloproteinase-2 expression in human fibroblasts. J Biomed Mater Res 39(3):437445

19. Eisenbarth E, Linez P, Biehl V, Velten D, Breme J, Hildebrand HF (2002) Cell orientation and cytoskeleton organisation on ground titanium surfaces. Biomol Eng 19(2-6):233-237

20. Eisenbarth E, Meyle J, Nachtigall W, Breme J (1996) Influence of the surface structure of titanium materials on the adhesion of fibroblasts. Biomaterials 17(14):1399-1403

21. Glass-Brudzinski J, Perizzolo D, Brunette DM (2002) Effects of substratum surface topography on the organization of cells and collagen fibers in collagen gel cultures. J Biomed Mater Res 61(4):608-618. doi:10.1002/jbm.10243

22. Oakley C, Brunette DM (1993) The sequence of alignment of microtubules, focal contacts and actin filaments in fibroblasts spreading on smooth and grooved titanium substrata. J Cell Sci 106(Pt 1): 343-354

23. Ramaglia L, Di Spigna G, Capece G, Sbordone C, Salzano S, Postiglione L (2015) Differentiation, apoptosis, and GM-CSF receptor expression of human gingival fibroblasts on a titanium surface treated by a dual acid-etched procedure. Clin Oral Investig 19: 2245-2253. doi:10.1007/s00784-015-1469-5

24. Satue M, Ramis JM, Monjo M (2015) Cholecalciferol synthesized after UV-activation of 7-dehydrocholesterol onto titanium implants inhibits osteoclastogenesis in vitro. J Biomed Mater Res A 103(7): 2280-2288. doi:10.1002/jbm.a.35364

25. Alfarsi MA, Hamlet SM, Ivanovski S (2014) The effect of platelet proteins released in response to titanium implant surfaces on macrophage pro-inflammatory cytokine Gene expression. Clin Implant Dent Relat Res. doi:10.1111/cid.12231

26. Hamlet S, Alfarsi M, George R, Ivanovski S (2012) The effect of hydrophilic titanium surface modification on macrophage inflammatory cytokine gene expression. Clin Oral Implants Res 23(5): 584-590. doi:10.1111/j.1600-0501.2011.02325.x

27. Hotchkiss KM, Reddy GB, Hyzy SL, Schwartz Z, Boyan BD, Olivares-Navarrete R (2015) Titanium surface characteristics, 
including topography and wettability, alter macrophage activation. Acta Biomater. doi:10.1016/j.actbio.2015.12.003

28. Refai AK, Textor M, Brunette DM, Waterfield JD (2004) Effect of titanium surface topography on macrophage activation and secretion of proinflammatory cytokines and chemokines. J Biomed Mater Res A 70(2):194-205. doi:10.1002/jbm.a.30075

29. Tan KS, Qian L, Rosado R, Flood PM, Cooper LF (2006) The role of titanium surface topography on J774A.1 macrophage inflammatory cytokines and nitric oxide production. Biomaterials 27(30): 5170-5177. doi:10.1016/j.biomaterials.2006.05.002

30. Thalji GN, Nares S, Cooper LF (2014) Early molecular assessment of osseointegration in humans. Clin Oral Implants Res 25(11): 1273-1285. doi:10.1111/clr.12266

31. Lee RS, Hamlet SM, Ivanovski S (2016) The influence of titanium surface characteristics on macrophage phenotype polarization during osseous healing in type I diabetic rats: a pilot study. Clin Oral Implants Res. doi:10.1111/clr.12979

32. Hotchkiss KM, Reddy GB, Hyzy SL, Schwartz Z, Boyan BD, Olivares-Navarrete R (2016) Titanium surface characteristics, including topography and wettability, alter macrophage activation. Acta Biomater 31:425-434. doi:10.1016/j.actbio.2015.12.003

33. Wang Y, Zhang Y, Jing D, Shuang Y, Miron RJ (2015) Enamel matrix derivative improves gingival fibroblast cell behavior cultured on titanium surfaces. Clin Oral Investig. doi:10.1007/ s00784-015-1558-5

34. Zhang Y, Wei L, Chang J, Miron RJ, Shi B, Yi S, Wu C (2013) Strontium-incorporated mesoporous bioactive glass scaffolds stimulating in vitro proliferation and differentiation of bone marrow stromal cells and in vivo regeneration of osteoporotic bone defects. J Mater Chem B 1(41):5711-5722. doi:10.1039/C3TB21047B

35. Zhang Y, Wei L, Miron RJ, Shi B, Bian Z (2014) Anabolic bone formation via a site specific bone targeting delivery system by interfering with semaphorin 4D expression. J Bone Min Res : Off J Am Soc Bone Min Res. doi:10.1002/jbmr.2322

36. Miron RJ, Hedbom E, Ruggiero S, Bosshardt DD, Zhang Y, Mauth C, Gemperli AC, lizuka T, Buser D, Sculean A (2011) Premature osteoblast clustering by enamel matrix proteins induces osteoblast differentiation through up-regulation of connexin 43 and $\mathrm{N}$ cadherin. PLoS One 6(8):e23375. doi:10.1371/journal.pone. 0023375

37. Miron RJ, Oates CJ, Molenberg A, Dard M, Hamilton DW (2010) The effect of enamel matrix proteins on the spreading, proliferation and differentiation of osteoblasts cultured on titanium surfaces. Biomaterials 31(3):449-460. doi:10.1016/j.biomaterials.2009.09.075

38. Anderson JM, Rodriguez A, Chang DT (2008). Foreign body reaction to biomaterials. In: Seminars in immunology, Elsevier, pp 86100

39. Ziats NP, Miller KM, Anderson JM (1988) In vitro and in vivo interactions of cells with biomaterials. Biomaterials 9(1):5-13

40. Thalji G, Cooper LF (2014) Molecular assessment of osseointegration in vitro: a review of current literature. Int J Oral Maxillofac Implants 29(2):e171-e199. doi:10.11607/jomi.te55

41. Chehroudi B, Ghrebi S, Murakami H, Waterfield JD, Owen G, Brunette DM (2010) Bone formation on rough, but not polished, subcutaneously implanted $\mathrm{Ti}$ surfaces is preceded by macrophage accumulation. J Biomed Mater Res A 93(2):724-737
42. Takebe J, Champagne CM, Offenbacher S, Ishibashi K, Cooper LF (2003) Titanium surface topography alters cell shape and modulates bone morphogenetic protein 2 expression in the J774A.1 macrophage cell line. J Biomed Mater Res A 64(2):207-216. doi:10. 1002/jbm.a.10275

43. Berglundh T, Gotfredsen K, Zitzmann NU, Lang NP, Lindhe J (2007) Spontaneous progression of ligature induced periimplantitis at implants with different surface roughness: an experimental study in dogs. Clin Oral Implants Res 18(5):655-661

44. Rimondini L, Faré S, Brambilla E, Felloni A, Consonni C, Brossa F, Carrassi A (1997) The effect of surface roughness on early in vivo plaque colonization on titanium. J Periodontol 68(6):556-562

45. Degidi M, Artese L, Piattelli A, Scarano A, Shibli JA, Piccirilli M, Perrotti V, Iezzi G (2012) Histological and immunohistochemical evaluation of the peri-implant soft tissues around machined and acid-etched titanium healing abutments: a prospective randomised study. Clin Oral Investig 16:857-866. doi:10.1007/s00784-011-0574-3

46. Derks J, Schaller D, Hakansson J, Wennstrom JL, Tomasi C, Berglundh T (2016) Effectiveness of implant therapy analyzed in a Swedish population: prevalence of peri-implantitis. J Dent Res 95(1):43-49. doi:10.1177/0022034515608832

47. Tarnow DP (2016) Increasing prevalence of peri-implantitis: how will we manage? J Dent Res 95(1):7-8. doi:10.1177/ 0022034515616557

48. Koldsland OC, Scheie AA, Aass AM (2010) Prevalence of periimplantitis related to severity of the disease with different degrees of bone loss. J Periodontol 81(2):231-238. doi:10.1902/jop.2009. 090269

49. Albrektsson T, Dahlin C, Jemt T, Sennerby L, Turri A, Wennerberg A (2014) Is marginal bone loss around oral implants the result of a provoked foreign body reaction? Clin Implant Dent Relat Res 16(2):155-165. doi:10.1111/cid.12142

50. Trindade R, Albrektsson T, Tengvall P, Wennerberg A (2014) Foreign body reaction to biomaterials: on mechanisms for buildup and breakdown of osseointegration. Clin Implant Dent Relat Res. doi:10.1111/cid.12274

51. Trindade R, Albrektsson T, Wennerberg A (2015) Current concepts for the biological basis of dental implants: foreign body equilibrium and osseointegration dynamics. Oral Maxillofac Surg Clin North Am 27(2):175-183. doi:10.1016/j.coms.2015.01.004

52. Hachim D, LoPresti ST, Yates CC, Brown BN (2017) Shifts in macrophage phenotype at the biomaterial interface via IL-4 eluting coatings are associated with improved implant integration. Biomaterials 112:95-107. doi:10.1016/j.biomaterials.2016.10.019

53. Lee CH, Kim YJ, Jang JH, Park JW (2016) Modulating macrophage polarization with divalent cations in nanostructured titanium implant surfaces. Nanotechnology 27:085101. doi:10.1088/09574484/27/8/085101

54. Vasconcelos DP, Costa M, Amaral IF, Barbosa MA, Aguas AP, Barbosa JN (2015) Development of an immunomodulatory biomaterial: using resolvin D1 to modulate inflammation. Biomaterials 53:566-573. doi:10.1016/j.biomaterials.2015.02.120

55. Vasconcelos DP, Costa M, Amaral IF, Barbosa MA, Aguas AP, Barbosa JN (2015) Modulation of the inflammatory response to chitosan through M2 macrophage polarization using proresolution mediators. Biomaterials 37:116-123. doi:10.1016/j. biomaterials.2014.10.035 